\title{
naverasancue \\ FIGO classification for the clinical diagnosis of placenta accreta spectrum disorders
}

\author{
Eric Jauniaux ${ }^{1, *}$, Diogo Ayres-de-Campos ${ }^{2}$, Jens Langhoff-Roos ${ }^{3}$, Karin A Fox ${ }^{4}$, Sally Collins ${ }^{5,6}$; \\ FIGO Placenta Accreta Diagnosis and Management Expert Consensus Panela
}

\author{
${ }^{1}$ EGA Institute for Women's Health, Faculty of Population Health Sciences, University College \\ London, London, UK \\ ${ }^{2}$ Medical School, University of Lisbon, Santa Maria Hospital, Lisbon, Portugal \\ ${ }^{3}$ Department of Obstetrics, Rigshospitalet, University of Copenhagen, Denmark \\ ${ }^{4}$ Division of Maternal-Fetal Medicine, Department of Obstetrics and Gynecology, Baylor College of \\ Medicine, Houston, Texas, USA \\ ${ }^{5}$ Nuffield Department of Women's and Reproductive Health, University of Oxford, Oxford, UK \\ ${ }^{6}$ Fetal Medicine Unit, John Radcliffe Hospital, Oxford, UK
}

\author{
*Correspondence \\ Eric Jauniaux, EGA Institute for Women's Health, Faculty of Population Health Sciences, University \\ College London, London, UK. \\ Email: e.jauniaux@ucl.ac.uk
}

ineveloped by the FIGO Safe Motherhood and Newborn Health Committee, coordinated by Eric Jauniaux.

$\star$ The views expressed in this document reflect the opinion of the individuals and not necessarily those of the institutions that they represent.

aFIGO Placenta Accreta Diagnosis and Management Expert Consensus Panel members are listed at the end of the paper.

\begin{abstract}
Placenta accreta spectrum is impacting maternal health outcomes globally and its prevalence is likely to increase. Maternal outcomes depend on the identification of the condition before or during delivery and, in particular, on the differential diagnosis between its adherent and invasive forms. However accurate estimation of its prevalence and outcome is currently problematic because of the varying use of clinical criteria to define it at birth and the lack of detailed pathologic examination in most series. Adherence to this new International Federation of Gynecology and Obstetrics (FIGO) classification should improve future systematic reviews and meta-analysis and provide more accurate epidemiologic data which are essential to develop new management strategies.
\end{abstract}

KEYWORDS: Accreta; Classification; Increta; Percreta; Placenta

Synopsis: Accurate identification and reporting of the different grades of placenta accreta spectrum are essential to the development of management strategies for this complex obstetric complication.

\begin{abstract}
1 INTRODUCTION
Irving and Hertig are credited for having published, in 1937, the first cohort study of placenta accreta in the international literature [1]. Their article included comprehensive clinical and histopathologic descriptions of 20 cases, and a literature review of 86 cases published before 1935 . All these cases were described as 'adherenta', which the authors characterized clinically as a placenta adherent to the uterine wall without easy separation and/or bleeding from the placental bed, and histologically as absence of decidual layer/Nitabuch layer between the placenta and myometrium. These diagnostic criteria were not new at the time and had been in use since the mid-1920s, including by the authors of case reports with histological evidence of villous invasion of the myometrium [2].
\end{abstract}

\section{HISTORICAL PERSPECTIVE}

Predisposing factors identified in the 1920s and 1930s were previous manual removal of placenta and/or "vigorous" uterine curettage. Only one of the 20 patients included in the Irving and Hertig series 
had a previous cesarean delivery [1]. Similarly, in their review of the previous 86 case reports, only one woman had a prior cesarean delivery. Before the development of antibiotics, damage to the uterine wall after uterine curettage or manual removal of the placenta was often aggravated by endometritis. This likely resulted in scar tissue forming focally within the superficial myometrium, which is not comparable to the extensive myometrial scar caused by multiple cesarean deliveries [4-6]. The low incidence of full-thickness myometrial scarring may explain why very few cases of invasive placentation were reported before the 1950s, when cesarean deliveries became safer and therefore increasingly common. There is now compelling epidemiological evidence that accreta placentation has become essentially an iatrogenic condition, secondary to the modern-era cesarean section epidemic $[3,4]$. In early pathologic studies, the distribution of adherent placenta accreta was found to be $69.5 \%$ whereas invasive placenta accreta represented $30.5 \%$ of all cases accreta placentation, respectively [3]. The incidence of invasive cases has increased in the last two decades, but accurate data are limited due to wide variation in the methodology used in cohort studies.

Placenta accreta was redefined in the mid-1960's by Lukes et al. [7] as a spectrum of abnormal placentation disorders. These disorders include placenta adherenta or vera also referred to as placenta creta by pathologists, in which the villi are attached directly to the surface of the myometrium without invading it; placenta increta, where the villi penetrate deeply into the myometrium up to the uterine serosa and placenta percreta, where the invasive villous tissue penetrates through the uterine serosa and may reach the surrounding pelvic tissues, vessels and organs. They also showed that different grades of the placenta accreta spectrum (PAS) can co-exist in the same specimens and that an accreta area can be focal or extended (diffuse). This remains the most comprehensive description of placenta accreta published so far, and was largely incorporated into the recent International Federation of Gynecology and Obstetrics (FIGO) guidelines [3] and other publications [6].

\section{DIAGNOSING PAS}

Similar to other clinical conditions, histopathology is now widely considered as the gold standard modality recommended to confirm clinical diagnosis of PAS, but it is often unavailable in adherent accreta or conservatively managed cases [3]. Moreover, unlike cancer staging, retrospective clinical and/or pathological grading of PAS has no direct long-term impact on the life of the patient. All these aspects may explain the apparent lack of interest in accurately differentiating between adherent and invasive forms, both by clinicians and pathologists and/or the lack of trained perinatal pathologists in many centers. As a result, the 1920-1930's criteria, based mostly on adherent cases, continued to be used by several authors of cohort studies. However, this can lead to misleading conclusions, as adherent and invasive accreta placentation have very different outcomes and require different management. To compound this, although $80 \%-90 \%$ of prenatally diagnosed PAS are managed surgically [8], around half of the authors fail to report the extent of villous attachment or invasion after peripartum hysterectomy [9].

Recent variants of the classical clinical description of PAS often include criteria such as a "difficult manual, piecemeal removal of the placenta"; "absence of spontaneous placental separation 20-30 minutes after birth despite active management, including bimanual massage of the uterus, use of oxytocin and controlled traction of the umbilical cord"; "retained placental fragment requiring curettage after vaginal birth"; and "heavy bleeding from the placentation site after removal of the placenta during cesarean delivery" [10-13]. This has resulted in a multitude of different clinical criteria, which can be easily confused with non-accreta placental retention and secondary uterine atony. With so many different criteria all purporting to represent PAS, but without any attempt to differentiate between adherent and invasive forms, it is unsurprising that there is a wide variation in the reported prevalence over the last 30 years.

Adding to the confusion is the wide heterogeneity in terminology used to describe the different grades of accreta placentation, including "placental adhesive disorders", "abnormal placental adherence", "advanced invasive placentation", and "abnormal myometrial invasion" [9,14]. A recent popular label used by clinicians reporting on the prenatal diagnosis of PAS has been "morbidly adherent placenta", which was used in the 19th century to describe placental retention. It has been recently used in WHO's 10th revision of the International Statistical Classification of Diseases (ICD-10) (www.who.int/classifications/icd) and has led to some exotic translation such as "the pernicious placenta" recently used by Chinese authors in both local and international journals $[15,16]$. This point also highlights the limited impact of accreta placentation research on the general scientific literature, as leading medical journals are unlikely to publish articles on diseases that do not have universally accepted diagnostic criteria and unequivocal terminology. Each of the other terminologies used so far are suboptimal and exclusive as they do not describe the different grades of PAS-i.e., "adherent", 
which does not include the invasive grades increta and percreta, and "invasive", which can be confused with gestational trophoblastic disease and, in particular, invasive intra-uterine choriocarcinoma.

Consequences reach far beyond a simple debate on what is the most adequate terminology.

So far, the lack of use of standardized clinical criteria for the diagnosis of the condition at birth and the histopathologic differential diagnosis between adherent and invasive accreta placentation has led to wide heterogeneity between studies for all epidemiologic and outcome parameters [18].

Distinguishing between adherent and invasive forms of accreta has a direct impact on the accurate evaluation of epidemiology, on improving the understanding of the underlying pathology and, most importantly, on the development of better management strategies. In addition, labelling cases of placenta retention as accreta or morbidly adherent leads to overdiagnosis, which can influence treatment decision leading to overtreatment and diagnosis related anxiety for many patients.

\section{PAS GRADING AND CLASSIFICATION}

The process of clarifying the reporting data on placenta accreta in the international literature started recently with the development of a grading system for the clinical diagnosis of PAS [19]. The classification presented in Box 1 was developed from this grading scheme, and reviewed by the members of the FIGO Placenta Accreta Spectrum Disorders Diagnosis and Management Expert Consensus Panel [20]. Of note, it refers to a classification and not a staging system, to differentiate it from the terminology used for cancer. As an example, for the use of the classification, we have summarized the recommendations of the recent FIGO guidelines for the conservative [21] and nonconservative surgical management [22] of PAS according to the grade of accreta invasiveness defined in the present classification (Box S1).

The accreta placentation process has an impact on both the anatomy of a portion of the placenta and on the development of the surrounding deep uterine circulation [6]. The accreta area will not spontaneously deliver at birth and any attempt in doing so may result in rapidly uncontrollable bleeding from the deep uterine vessels or the neovascularisation around the accreta area. The deeper and larger the accreta area inside the uterine wall, the higher the risks of severe hemorrhagic complications and need to perform an emergency hysterectomy. To avoid unnecessary complex surgical procedure, clinicians should differentiate between placenta percreta and a so-called uterine window, which is an area of cesarean scar dehiscence with normal placentation underneath (or sometime seen poking through). In the latter, the surrounding uterine tissue appears relatively normal with no neovascularity or placental bulge. If the placenta is eventually delivered manually in whole or in pieces, it is unlikely to have been accreta. The manual removal of a non-accreta retained placenta can also be associated with severe hemorrhage due to secondary uterine atony, but in these cases, conservative management techniques such as compressive sutures and intrauterine balloon are often successful in controlling the bleeding. These cases should not be reported as successful management of PAS.

\section{REPORTING DATA ON PAS}

It is pivotal to improve the accuracy of PAS diagnosis in the international literature, and for this purpose we also propose reporting guidelines, which include a standardized basic dataset for future clinical research and to allow comparison between centers with different management strategies (Box 2). This protocol does not replace the general EQUATOR network guidelines (https://www.equatornetwork.org/), such as the PRISMA guideline for systematic reviews, but rather it serves to elevate the international discourse about PAS to a scientific caliber that matches the importance of the disease in maternal health globally. Adherence to this new FIGO classification will improve future systematic reviews and meta-analysis and provide more accurate epidemiologic data which are essential to improve clinical outcomes.

\section{CONSENSUS PANEL}

Greg Duncombe (Australia and New Zealand), Philipp Klaritsch (Germany), Frédéric Chantraine (Belgium), John Kingdom (Canada), Lene Grønbeck (Denmark), Kristiina Rull (Estonia), Minna Tikkanen (Finland), Loïc Sentilhes (France), Tengiz Asatiani (Georgia), Wing-Cheong Leung (Hong Kong), Taghreed Alhaidari (Iraq), Donal Brennan (Ireland), Muhieddine Seoud (Lebanon), Ahmed Mahmoud Hussein (Egypt), Ravindran Jegasothy (Malaysia), Kamal Nusrat Shah (Pakistan), Dorota Bomba-Opon (Poland), Corinne Hubinont (Belgium), Priya Soma-Pillay (South Africa), Nataša Tul Mandić (Slovenia), Pelle Lindqvist (Sweden), Berglind Arnadottir (Sweden), Irene Hoesli (Switzerland), Rafael Cortez (Venezuela).

CONFLICTS OF INTEREST The authors have no conflicts of interest. 


\section{References}

1. Irving C, Hertig AT. A study of placenta accreta. Surgery, Gynecol Obstet. 1937;64:178-200.

2. Forster DS. A case of placenta accreta. Can Med Assoc J. 1927;17:204-7.

3. Jauniaux E, Chantraine F, Silver RM, Langhoff-Roos J; FIGO Placenta Accreta Diagnosis and Management Expert Consensus Panel. FIGO consensus guidelines on placenta accreta spectrum disorders: Epidemiology. Int J Gynaecol Obstet. 2018;140:265-73.

4. Jauniaux E, Jurkovic D. Placenta accreta: pathogenesis of a 20th century iatrogenic uterine disease. Placenta. 2012;33:244-51.

5. Jauniaux E, Bhide A, Burton GJ. Pathophysiology of accreta. In: Silver, R. ed. Placenta accreta syndrome. Portland: CRC Press; 2017:13-28.

6. Jauniaux E, Collins SL, Burton GJ. Placenta accreta spectrum: Pathophysiology and evidence-based anatomy for prenatal ultrasound imaging. Am J Obstet Gynecol. 2018;218:75-87.

7. Luke RK, Sharpe JW, Greene RR. Placenta accreta: The adherent or invasive placenta. Am J Obstet Gynecol. 1966;95:660-8.

8. Jauniaux E, Bhide A. Prenatal ultrasound diagnosis and outcome of placenta previa accreta after caesarean delivery: a systematic review and meta-analysis. Am J Obstet Gynecol 2017;217:27-36.

9. Jauniaux E, Collins SL, Jurkovic D, Burton GJ. Accreta placentation. A systematic review of prenatal ultrasound imaging and grading of villous invasiveness. Am J Obstet Gynecol. 2016; 215:712-21.

10. Gielchinsky Y, Rojansky N, Fasouliotis SJ, Ezra Y. Placenta accreta--summary of 10 years: a survey of 310 cases. Placenta. 2002;23:210-4.

11. Wu S, Kocherginsky M, Hibbard JU. Abnormal placentation: twenty-year analysis. Am J Obstet Gynecol. 2005;192:1458-61.

12. Umezurike CC, Nkwocha G. Placenta accreta in Aba, south eastern, Nigeria. Niger J Med. 2007;16:219-22.

13. Seet EL, Kay HH, Wu S, Terplan M. Placenta accreta: depth of invasion and neonatal outcomes. J Matern Fetal Neonatal Med. 2012;25:2042-5.

14. Collins SL, Chantraine F, Morgan TK, Jauniaux E. Abnormally adherent and invasive placenta: A spectrum disorder in need of a name. Ultrasound Obstet Gynecol. 2018;51:165-6.

15. McDonald KN. How to prevent septicaemia in cases of morbidly adherent placenta. BMJ. 1885;1:779-80.

16. Huang $S$, Xia A, Jamail G, Long $M$, Cheng $C$. Efficacy of temporary ligation of infrarenal abdominal aorta during cesarean section in pernicious placenta previa. Zhong Nan Da Xue Xue Bao Yi Xue Ban. 2017;42:313-19.

17. Dai MJ, Jin GX, Lin JH, Zhang Y, Chen YY, Zhang XB. Pre-cesarean prophylactic balloon placement in the internal iliac artery to prevent postpartum hemorrhage among women with pernicious placenta previa. Int J Gynaecol Obstet. 2018;142;315-20.

18. Jauniaux E, Bunce C, Grønbeck L, Langhoff-Roos J. Prevalence and main outcomes of placenta accreta spectrum: a systematic review and metaanalysis. Am J Obstet Gynecol 2019;220: in press.

19. Collins SL, Stevenson GN, Al-Khan A, Illsley NP, Impey L, Pappas L, et al. ThreeDimensional Power Doppler Ultrasonography for Diagnosing Abnormally Invasive Placenta and Quantifying the Risk. Obstet Gynecol. 2015;126:645-53.

20. Jauniaux E, Ayres-de-Campos D; for the FIGO Placenta Accreta Diagnosis and Management Expert Consensus Panel. FIGO consensus guidelines on placenta accreta spectrum disorders: Introduction. Int J Gynecol Obstet 2018; 140:261-4.

21. Sentilhes L, Kayem G, Chandraharan E, Palacios-Jaraquemada J, Jauniaux E; FIGO Placenta Accreta Diagnosis and Management Expert Consensus Panel. FIGO consensus guidelines on placenta accreta spectrum disorders: Conservative management. Int J Gynaecol Obstet. 2018;140:291-8.

22. Allen L, Jauniaux E, Hobson S, Papillon-Smith J, Belfort MA; FIGO Placenta Accreta Diagnosis and Management Expert Consensus Panel. FIGO consensus guidelines on placenta accreta spectrum disorders: Non-conservative surgical management. Int J Gynaecol Obstet. 2018;140:281-90.

SUPPORTING INFORMATION Additional Supporting Information may be found online in the supporting information tab for this article. 


\section{Box 1 General classification of placenta accreta spectrum ${ }^{a}$}

\section{Grade 1: Abnormal adherent placenta (placenta adherenta or creta)}

Clinical criteria

- At vaginal delivery

- No separation with synthetic oxytocin and gentle controlled cord traction

- Attempts at manual removal of the placenta results in heavy bleeding from the placenta implantation site requiring mechanical or surgical procedures

- If laparotomy is required

- Same as above

- Macroscopically, the uterus shows no obvious distension over the placental bed (placental "bulge"), no placental tissue is seen invading through the surface of the uterus, and there is no or minimal neovascularity

Histologic criteria

- Microscopic examination of the placental bed samples from hysterectomy specimen shows extended areas of absent decidua between villous tissue and myometrium with placental villi attached directly to the superficial myometrium

- The diagnosis cannot be made on just delivered placental tissue nor on random biopsies of the placental bed

\section{Grade 2: Abnormally invasive placentation (placenta increta)}

Clinical criteria

- At laparotomy

- Abnormal macroscopic findings over the placental bed: bluish/purple colouring, distension (placental "bulge")

- Significant amounts of hypervascularity (dense tangled bed of vessels or multiple vessels running parallel craniocaudially in the uterine serosa)

- No placental tissue seen to be invading through the surface of the uterus.

- Gentle cord traction results in the uterus being pulled inwards without separation of the placenta (so-called the dimple sign)

Histologic criteria

- Hysterectomy specimen or partial myometrial resection of the increta area shows placental villi within the muscular fibers and sometimes in the lumen of the deep uterine vasculature (Radial or Arcuate arteries)

Grade 3: Abnormally invasive placentation (placenta percreta)

Grade 3a: Limited to the uterine serosa

Clinical criteria

- At laparotomy

- Abnormal macroscopic findings on uterine surface (as above) and placental tissue seen to be invading through the surface of the uterus (serosa)

- No invasion into any other organ, including the posterior wall of the bladder (a clear surgical plane can be identified between the bladder and uterus)

- Histologic criteria

- Hysterectomy specimen showing villous tissue within or breaching the uterine serosa

Grade 3b: With urinary bladder invasion

Clinical criteria

- At laparotomy

- Same as 3a

- Placental villi are seen to be invading into the bladder but no other organs

- Clear surgical plane cannot be identified between the bladder and uterus

Histologic criteria

- Hysterectomy specimen showing villous tissue breaching the uterine serosa and invading the bladder wall tissue or urothelium 
Grade 3c: With invasion of other pelvic tissue/organs

Clinical criteria

- At laparotomy

- Same as 3a

- Placental villi are seen to be invading into the broad ligament, vaginal wall, pelvic sidewall or any other pelvic organ (with or without invasion of bladder)

Histologic criteria

- Hysterectomy specimen showing villous tissue breaching the uterine serosa and invading pelvic tissues/organs

aFor the purposes of this classification, "uterus" includes the uterine body and uterine cervix

\section{Box 2: Basic dataset for reporting on placenta accreta spectrum}

\section{Background population}

- Institution-based study

- Display referred cases and cases from local catchment area in separate data sets

- Description of background population and cases including number of births, mode of delivery, parity, local $C D$ rate (stratified by numbers of prior deliveries and numbers of prior $C D$ )

- Regional/network/national-based study

- Description of local background population including number of births, mode of delivery, parity, $C D$ rates (stratified by numbers of prior deliveries and numbers of prior $C D$ ) for referred cases and local cases.

Description of standardized criteria used for prenatal diagnosis

- Ultrasound signs of placenta accreta spectrum, including placental location

- MRI signs of PAS including surface area and depth

\section{Management strategy}

- Intended mode of management: vaginal delivery, scheduled CD, hysterectomy (primary or delayed), focal myometrial resection, leaving the placenta in situ

- Actual mode of management: vaginal delivery, scheduled CD, emergent $C D$, focal myometrial resection, hysterectomy (primary or delayed), leaving the placenta in situ

\section{Confirmation of diagnosis:}

- Clinical diagnostic criteria and confirmed histopathological diagnosis when possible

- The final diagnosis (clinical, histopathological) should be clearly stated and made according to the classification in Box 1

Abbreviations: $\mathrm{CD}$, caesarean delivery; MRI, magnetic resonance imaging.

Box S1. Example of management for placenta accreta spectrum using the new classification presented in Box 1 